\title{
Compensation effect analysis in DIE method for through-casing measuring formation resistivity
}

\author{
Chen Qing $^{1}, \quad$ David Pardo ${ }^{2}$, Li Hong-bin ${ }^{1}, \quad$ Wang Fu-rong ${ }^{3}$ \\ 1. CEEE of Huazhong University of Science and Technology, 430074Wuhan, China \\ 2. Department of Applied Mathematics, Statistics, and Operational Research at UPV/EHU, and IKERBASQUE, Basque Foundation for Sciences, Bilbao, Spain \\ 3. China University of Geosciences, 430074 Wuhan, China
}

\begin{abstract}
Double-Injection-Electrodes (DIE) model and its compensation arithmetic method has been proven to be very useful for eliminating the errors caused by electrode-scale mechanical tolerances in formation resistivity measurement through metal case. In this paper, we found that even minor casing joint or casing corrosion may deteriorate the measurement accuracy. Based on theoretical analysis and self-adaptive goal oriented $h p$-Finite Element (FE) simulations, the compensation effects of DIE model were estimated. The calculated results from DIE model are always close to the real formation resistivity no matter the metal casing is ideal or not. Meanwhile, large errors occur in Single-Injection-Electrode (SIE) model, where the calculated formation resistivity may provide negative numbers when casing joint or casing corrosion exists. The Double-Injection-Electrode (DIE) model is predicted to have good compensation effects to many non-ideal situations with uneven metal casing besides electrode-scale mechanical tolerances.
\end{abstract}

\section{Introduction}

Acquisition of through-casing resistivity (TCR) measurement was first proposed by Alpin in 1939 [1]. He stated that when current is injected into a casing, the voltage differences on the casing well are highly influenced by the formation resistivities. Thus, formation resistivity could be inferred by measuring the voltage differences.

Due to the weak voltage signals below $1 \mu \mathrm{V}$ obtained in TCR measurements and the limited technology existing at the time for measuring such a low voltage signals, Alpin's method could not be implemented at that time.

Several decades later, recent advances in weak signal processing and measurement technologies have allowed Alpin's method to be applied. The report on the field test of the prototype of the tool (Vail et al., 1995) was another important step towards the development of the technology. During the last two decades, the interest in electrical logging through casing has grown considerably, since resistivity estimations in cased wells is as topic of great importance for the logging industry.

Several studies have been performed concerning TCR measurements. In particular, vertical resolution of the measurement, effects caused by the cement sheath, casing inhomogeneities, and the finite length of the casing have been studied by Schenkel (1990), Kaufman and Wightman (1993), Schenkel and Morrison (1994), Tabarovsky et al.
(1994), Zinger et al. (1994), and Singer et al. (1995 and 1998) [2]-[10]. Based on Kaufman measurement mode, various attempts have been made to build systems for logging formation resistivity in cased boreholes [11]-[12] .

Kaufman's theory has always been seemed as the technology basis of through casing resistivity measurement. However, Kaufman's Single-Injection-Electrode (SIE) model and relative arithmetic may present some disadvantages when applied to complex TCR models. In reference [13], Chen and Pardo (2010) found that the measurement errors are very sensitive to the mechanical tolerances. In order to reduce the errors, a Double-Injection-Electrode (DIE) model was presented to correct the SIE model. While most analytical methods cannot be applied to complex geometries, simulation of TCR measurements via numerical methods is rather challenging due to the high electrical conductivity contrast and small thickness of casing [14]-[21]. Utilizing a 2-D axially symmetric numerical method based on a self-adaptive goal oriented $h p$-finite-element method (FEM), Chen and Pardo proved that, when considering the SIE model, it is still not certain that the error can be controlled below $5 \%$ even if the mechanical tolerance is below $0.01 \%$. However, when using the DIE model accompanied with compensation arithmetic method, even a $10 \%$ mechanical tolerance has very little impact on the results (nearly $2 \%$ ).

In this paper, we extend the analysis and study of the DIE method to the case of casing imperfections. In particular, the errors caused by casing joint and casing corrosion besides mechanical tolerance are calculated through theoretical analysis and self-adaptive goal oriented $h p$-finite-element method (FEM) simulations, through which the compensation effects of symmetrical arithmetic method derived from DIE model are estimated correctly. Moreover, it is predicted that DIE model will decrease the errors caused by other non-ideal situations of uneven metal casing besides casing joint and casing corrosion.

\section{SIE and DIE models}

\section{A. SIE model}

For the SIE model, the casing is assumed to be a uniform and highly conductive steel pipe with an infinite length. The formation is assumed to be a homogeneous medium around the casing. The leakage current is known to be perpendicular to the casing. As shown in Fig. 1, the current is injected into the casing from electrode $\mathrm{A}$, and the 
formation apparent resistivity around point $\mathrm{D}$, namely $\rho_{S I E}$, is estimated as:

$$
\begin{aligned}
& \rho_{\mathrm{SIE}}=(\Delta z)^{2} \frac{U_{D}}{\Delta^{2} U} r_{c} \\
& \Delta^{2} U=U_{C D}-U_{D E}
\end{aligned}
$$

where $r_{c}$ is the casing resistance per meter, $\Delta z$ is the distance between two consecutive electrodes, and $U_{D}, U_{C D}$, and $U_{D E}$ are the voltage difference signals described in Fig.1. Notice that the computed result $\rho_{a}$ obtained from equation (1) is not the real resistivity of formation, but the formation resistance of the neighboring horizontal layer. The conversion factor from $\rho_{a}$ to the real formation resistivity depends upon the mechanical characteristics of the casing.

In order to estimate $\rho_{a}$, we need to calculate $r_{c}$ and measure $\left(U_{C D}-U_{D E}\right), \Delta \mathrm{z}$, and $U_{D}$. The quality of the approximation of $\rho_{a}$ is highly dependent upon the accuracy of the estimated steel casing resistance $r_{c}$. There exist two ways to calculate $r_{c}$.

One is a theoretical calculation method. Assuming known values of $\rho_{c}$ (steel casing resistivity), $a$ and $\Delta a$ (radius of casing and the thickness of casing, respectively), $r_{c}$ can be represented as:

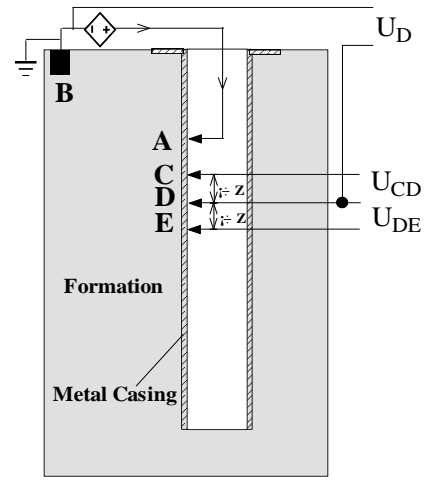

Fig.1 SIE model

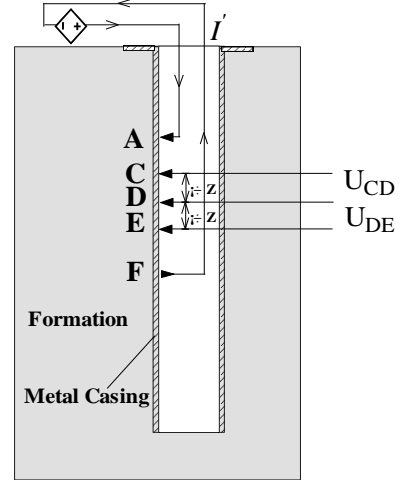

Fig.2 $r_{c}$ measurement in SIE model

$$
r_{c}=\rho_{c} \frac{1}{2 \pi a \Delta a}
$$

In equation (2), $\rho_{c}$ is sensitive to temperature change, and 100 degrees temperature variation will bring an excursion over $20 \%$ to $\rho_{c}$. Thus, in equation (2), the calculated $r_{c}$ can not reflect the real steel casing resistance, and the theoretical calculation method will inevitably produce a large error in resistivity measurement.

The second method to estimate $r_{c}$ is based on a practical measurement that is immune to temperature variations. In this method, the operation program becomes more complex, which lowers the logging efficiency. As shown in Fig. 2, the current $I$ is injected from electrode A and collected at electrode $\mathrm{B}$. Then, $r_{c}$ can be inferred from the following equation:

$$
r_{c}=\frac{U_{C D}^{\prime}}{I^{\prime} \Delta z}=\frac{U_{D E}^{\prime}}{I^{\prime} \Delta z}=\frac{U_{C D}^{\prime}+U_{D E}^{\prime}}{2 I^{\prime} \Delta z}
$$

Thus, the apparent formation resistivity in SIE model becomes:

$$
\rho_{\mathrm{SIE}}=\frac{\Delta z}{2 I^{\prime}} \frac{U_{D}\left(U_{C D}^{\prime}+U_{D E}^{\prime}\right)}{U_{C D}-U_{D E}}
$$

\section{B. DIE model}

As shown in Fig. 3, the Double-Injection-Electrodes (DIE) model differs from that of SIE in that an additional current injection electrode $\mathrm{F}$ is used, and point $\mathrm{D}$ is assumed to be the midpoint of $l_{A F}$. The current is injected from electrode $\mathrm{A}$ and electrode $\mathrm{F}$ alternately, and the injected currents are $I_{A}$ and $I_{F}$, respectively. When the current $I_{A}$ is injected from electrode $\mathrm{A}$, the voltage difference between electrode $\mathrm{C}$ and $\mathrm{D}$ is now denoted as $U_{A-C D}$ in order to distinguish it from $U_{F-C D}$, the voltage difference between electrodes $\mathrm{C}$ and $\mathrm{D}$ when the current $I_{F}$ is injected from electrode $\mathrm{F}$. The remaining symbols can be understood in the same way, as described in equation (5).

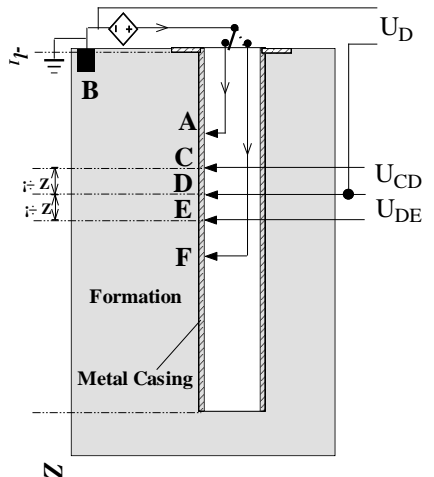

Fig.3 DIE model

$$
\begin{aligned}
& U_{A-C D}=U_{A-C}-U_{A-D} \\
& U_{F-C D}=U_{F-C}-U_{F-D} \\
& U_{A-D E}=U_{A-D}-U_{A-E} \\
& U_{F-D E}=U_{F-D}-U_{F-E} \\
& U_{A-C E}=U_{A-C D}+U_{A-D E} \\
& U_{F-C E}=U_{F-C D}+U_{F-D E} \\
& \Delta^{2} U_{A}=U_{A-C D}-U_{A-D E} \\
& \Delta^{2} U_{F}=U_{F-C D}-U_{F-D E}
\end{aligned}
$$

Without applying the connection method used in SIE model as shown in Fig. 2, now $r_{c}$ can be obtained directly: 


$$
r_{c}=\frac{\Delta^{2} U_{A}+U_{A-C E}}{2 I_{A} \Delta \mathrm{z}}+\frac{\Delta^{2} U_{F}-U_{F-C E}}{2 I_{F} \Delta \mathrm{z}}
$$

In the above equation, $\Delta^{2} U_{A}$ and $\Delta^{2} U_{F}$ provide the apparent compensations for the real steel casing resistance, through which the influence of the parallel formation resistance is eliminated, especially when the formation resistance is low.

In the innovative DIE model, the other two important parameters, $U_{D}$ and $\Delta^{2} U$, presented in equation (1) are defined as:

$$
\begin{aligned}
& U_{D}=\frac{U_{A-C E} U_{F-D}-U_{F-C E} U_{A-D}}{U_{A-C E}-U_{F-C E}} \\
& \Delta^{2} U=\frac{U_{A-C E} \Delta^{2} U_{F}-U_{F-C E} \Delta^{2} U_{A}}{U_{A-C E}-U_{F-C E}}
\end{aligned}
$$

Thus, the final formation apparent resistivity in DIE model becomes:

$$
\begin{aligned}
\rho_{\mathrm{DIE}}= & \frac{\Delta z}{2}\left(\frac{\Delta^{2} U_{A}+U_{A-C E}}{I_{A}}+\frac{\Delta^{2} U_{F}-U_{F-C E}}{I_{F}}\right) \\
& \cdot \frac{U_{A-C E} U_{F-D}-U_{F-C E} U_{A-D}}{U_{A-C E} \Delta^{2} U_{F}-U_{F-C E} \Delta^{2} U_{A}}
\end{aligned}
$$

\section{Theoretical analysis}

The object of the analysis is to determine the errors caused by casing joint and casing corrosion in SIE and DIE models. As shown in Fig.4, the metal casing is assumed to have an overall length $h$, an average radius $a$ and a thickness $\Delta a$. The injected current $I$ is located at electrode A or F. The leaking current distributes along the casing, and the total leaking current values of casing segments $h_{l}$, $h_{2}$ and $h_{3}$ are respectively $\Delta I_{1}, \Delta I_{2}$ and $\Delta I_{3}$. It is assumed that the conductivity of metal casing is more than $10^{9}$ times of formation conductivity, so the leaking current $\Delta I_{2}$ is considered distributed uniformly along the casing segment $h_{2}$.

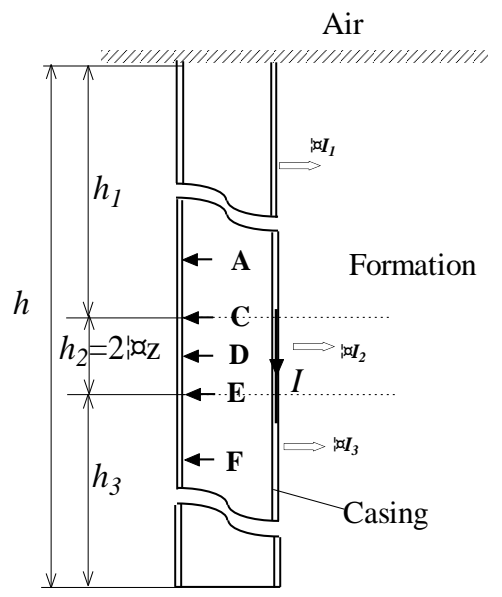

Fig.4 Currents distributed along the casing
When the current is injected from electrode $A$, the current flowing along the casing vertically at points $\mathrm{C}, \mathrm{D}$ and $\mathrm{E}$ can be described as

$$
\begin{aligned}
& I_{C}=\Delta I_{2}+\Delta I_{3} \\
& I_{D}=\frac{1}{2} \Delta I_{2}+\Delta I_{3} \\
& I_{E}=\Delta I_{3} \\
& U_{C D}=r_{C D}\left(\frac{3}{4} \Delta I_{2}+\Delta I_{3}\right) \\
& U_{D E}=r_{D E}\left(\frac{1}{4} \Delta I_{2}+\Delta I_{3}\right)
\end{aligned}
$$

If we assume:

$$
\begin{aligned}
& h_{2}<h \\
& \Delta I_{2}<<\Delta I_{1}+\Delta I_{2}
\end{aligned}
$$

Then, $U_{D}, \Delta I_{1}, \Delta I_{2}$ and $\Delta I_{3}$ are independent of the electrode where the current is injected (A or F). When the current is injected from electrode $\mathrm{F}$, the vertical currents along the casing are:

$$
\begin{aligned}
& I_{C}=-\Delta I_{1} \\
& I_{D}=-\Delta I_{1}-\frac{1}{2} \Delta I_{2} \\
& I_{E}=-\Delta I_{1}-\Delta I_{2} \\
& U_{C D}=-r_{C D}\left(\Delta I_{1}+\frac{1}{4} \Delta I_{2}\right) \\
& U_{C D}=-r_{C D}\left(\Delta I_{1}+\frac{3}{4} \Delta I_{2}\right)
\end{aligned}
$$

In absence of casing joints or corrosion, $r_{C D}$ (the casing resistance between electrode $\mathrm{C}$ and electrode $\mathrm{D}$ ) is equal to $r_{D E}$ (the casing resistance between $\mathrm{D}$ and electrode $\mathrm{E}$ ), that is:

$$
r_{C D}=r_{D E}=\Delta z \cdot r_{c}
$$

In SIE model, the formation resistivity $\rho_{S I E}$ can be calculated by substituting equations (9) and (12) into equation (1).

$$
\rho_{\mathrm{SIE}}=2 \Delta z \frac{U_{D}}{\Delta I_{2}}
$$

Substituting equations (9), (11), and (12) into equation (4), $\rho_{D I E}$ (the formation resistivity of DIE model) becomes:

$$
\rho_{\mathrm{DIE}}=2 \Delta z \frac{U_{D}}{\Delta I_{2}}
$$

There is no doubt that casing joints or corrosion will bring variations to $r_{C D}$ and $r_{C D}$.

If

$$
\begin{aligned}
& r_{C D}=K_{1} \cdot \Delta z \cdot r_{c} \\
& r_{D E}=K_{2} \cdot \Delta z \cdot r_{c}
\end{aligned}
$$


then, the formation resistivities in SIE model and DIE model can be calculated as:

$$
\begin{aligned}
& \rho_{\mathrm{SIE}}{ }^{*}=2 \Delta z \frac{\left(K_{1}+K_{2}\right) U_{D}}{\left(3 K_{1}-K_{2}\right) \Delta I_{2}+\left(K_{1}-K_{2}\right) \Delta I_{3}} \\
& \rho_{\mathrm{DIE}}{ }^{*}=\Delta z \frac{\left(K_{1}+K_{2}\right)^{2}}{2 K_{1} K_{2}} \frac{U_{D}}{\Delta I_{2}}
\end{aligned}
$$

The length of metal casing is more than 1000 times that of the casing radius, so the leaking current can be assumed to distribute nearly uniformly along the casing, that is to say $\Delta I_{1}, \Delta I_{2}$ and $\Delta I_{3}$ are proportional to $h_{1}, h_{2}$ and $h_{3}$ respectively. From equations (13), (14) and (16), we derive the relative errors caused by casing resistance variations in SIE and DIE models:

$$
\begin{aligned}
& e_{\mathrm{SIE}}=\frac{\rho_{\mathrm{SIE}}{ }^{*}-\rho_{\mathrm{SIE}}}{\rho_{\mathrm{SIE}}}=\frac{\left(K_{2}-K_{1}\right)\left(2 h_{2}+h_{3}\right)}{\left(3 K_{1}-K_{2}\right) h_{2}+\left(K_{1}-K_{2}\right) h_{3}} \\
& e_{D I E}=\frac{\rho_{\mathrm{DIE}}{ }^{*}-\rho_{\mathrm{DIE}}}{\rho_{\mathrm{DIE}}}=\frac{\left(K_{1}-K_{2}\right)^{2}}{4 K_{1} K_{2}}
\end{aligned}
$$

Equation (17) indicates that the errors caused by casing joint or corrosion in SIE model are much more sensitive to the coefficients $K_{1}$ and $K_{2}$ than those in DIE model. Moreover, because $h_{3}$ is always much longer than $h_{2}$, it is not certain that $e_{S I E}$ will be limited below $5 \%$ even if the difference between $K_{1}$ and $K_{2}$ is less than $0.1 \%$.

\section{IV $h p$-FEM Simulations}

In this section, we simulate SIE model and DIE model at DC. Substituting the simulation results to equations (4) and (8), we can estimate the formation resistivity. By comparing those numerical results against the real formation resistivity assumed before simulations, the performance of SIE and DIE arithmetic methods are estimated.

Simulation of TCR measurements via numerical methods is rather challenging due to the high electrical conductivity contrast and small thickness of casing [13]-[21]. Here, we utilize a 2-D axially symmetric numerical method based on a self-adaptive goal oriented $h p$-finite-element method (FEM) that accurately simulates such logging measurements. This method constructs automatically an optimal grid with varying element sizes $h$ and polynomials orders of approximation $p$ throughout the computational grid, and it produces high-accuracy solutions that we employ to compare the performance of the SIE model vs. the DIE model..

\section{A. Ideal casing}

For SIE model, there are two steps to estimate the formation resistivity:

(1) one is to measure the casing resistance $r_{c}$, as shown in Fig.2. The relative parameters are described as:

$$
\begin{aligned}
& h=100 \mathrm{~m} \\
& h_{D}=50 \mathrm{~m} \\
& l_{A D}=l_{D F}=1.5 \mathrm{~m} \\
& \Delta z=0.5 \mathrm{~m} \\
& a=0.15 \mathrm{~m} \\
& \Delta a=0.01 \mathrm{~m}
\end{aligned}
$$

In the above Equation: 1.) $h, a$ and $\Delta a$ are the length, average radius, and thickness of the casing; 2.) $h_{D}$ is the distance from the ground to electrode $\mathrm{D} ; 3$.) $l_{A D}$ and $l_{D B}$ are the distances between $\mathrm{A}$ and $\mathrm{D}$ and between $\mathrm{D}$ and $\mathrm{F}$; 4.) $\Delta z$ is the length unit of electrode-scale and it is equal to $0.5 \mathrm{~m}$. The casing resistivity $\rho_{c}$, the borehole resistivity $\rho_{b}$ and the formation resistivity $\rho$ are respectively assumed to be $1 \times 10^{-6} \Omega \cdot \mathrm{m}, 1 \Omega \cdot \mathrm{m}$ and $100 \Omega \cdot \mathrm{m}$. The injected current $I^{\prime}$ is assumed to be $100 \mathrm{~A}$, and the potential differences $U_{C D}^{\prime}$ and $U_{D E}^{\prime}$ are calculated through $h p$ FEM simulations:

$$
\begin{aligned}
& I^{\prime}=100 \mathrm{~A} \\
& U_{C D}^{\prime}=0.002858 \mathrm{~V} \\
& U_{D E}^{\prime}=0.002849 \mathrm{~V}
\end{aligned}
$$

(2) the second step is to compute the potential $U_{D}$ and second potential difference $\Delta^{2} U$, as shown in Fig.1. All the parameters are the same as those described above. When a current $I$ with $100 \mathrm{~A}$ is injected from electrode A, the relative potentials are:

$$
\begin{aligned}
& I=100 \mathrm{~A} \\
& U_{C}=1.232315 \mathrm{~V} \\
& U_{D}=1.229457 \mathrm{~V} \\
& U_{E}=1.226630 \mathrm{~V}
\end{aligned}
$$

Substituting the results into equation (4), $\rho_{l}$, the calculated formation resistivity of SIE model is obtained, as shown in TABLE I.

In DIE model, all the parameters can be measured in one step. As shown in Fig.3, a current with value of $100 \mathrm{~A}$ is injected from electrode A and F alternatively, and all the relative potentials can be calculated through simulations:

$$
\begin{aligned}
& I_{A}=I_{F}=100 \mathrm{~A} \\
& U_{A-C}=1.232315 \mathrm{~V} \\
& U_{A-D}=1.229457 \mathrm{~V} \\
& U_{A-E}=1.226630 \mathrm{~V} \\
& U_{F-C}=1.226133 \mathrm{~V} \\
& U_{F-D}=1.228712 \mathrm{~V} \\
& U_{F-E}=1.231321 \mathrm{~V}
\end{aligned}
$$

Substituting the results into equation (8), $\rho_{2}$, the calculated formation resistivity of DIE model is obtained, as shown in TABLE I. 


\section{B. Presence of casing joints}

Casing joint is a popular method that accounts for possible imperfections on a metallic casing. We consider a casing joint that has wider thickness compared with the main part of casing, as shown in Fig. 5. Taking no account of the contact resistance, the joint located between electrode $\mathrm{C}$ and electrode $\mathrm{D}$ is considered to have a resistivity equal to the casing resistivity $1 \times 10^{-6} \Omega \cdot \mathrm{m}$.

According to the simulation procedures described above, the potentials at the measurement electrodes are computed.

For SIE model, the relative potentials are:

$$
\begin{aligned}
& I^{\prime}=100 \mathrm{~A} \\
& U_{C D}^{\prime}=0.002607 \mathrm{~V} \\
& U_{D E}^{\prime}=0.002831 \mathrm{~V} \\
& I=100 \mathrm{~A} \\
& U_{C}=1.232211 \mathrm{~V} \\
& U_{D}=1.229604 \mathrm{~V} \\
& U_{E}=1.226776 \mathrm{~V}
\end{aligned}
$$

For DIE model, the relative potentials are:

$$
\begin{aligned}
& I_{A}=I_{F}=100 \mathrm{~A} \\
& U_{A-C}=1.232211 \mathrm{~V} \\
& U_{A-D}=1.229046 \mathrm{~V} \\
& U_{A-E}=1.226776 \mathrm{~V} \\
& U_{F-C}=1.226280 \mathrm{~V} \\
& U_{F-D}=1.228634 \mathrm{~V} \\
& U_{F-E}=1.231244 \mathrm{~V}
\end{aligned}
$$

Based on equations (4) and (8), $\rho_{3}$ and $\rho_{4}$, formation resistivities for the case of a casing joint from SIE and DIE models, respectively can be easily calculated, as shown in TABLE I.

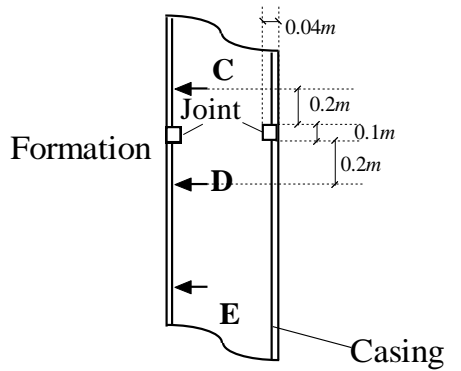

Fig.4 Casing with joint

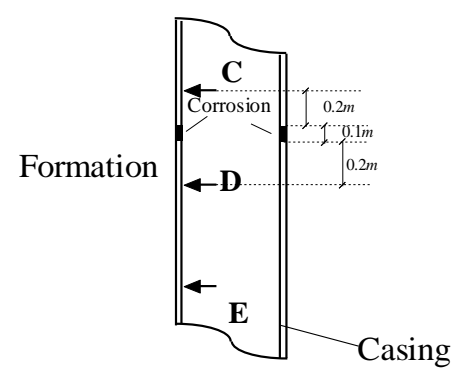

Fig.5 Casing with corrosion

\section{Presence of casing corrosion}

Typically, due to corrosion and damage, a casing is not uniform in resistivity along the borehole and, hence, it contains zones with different conductivities. Fig. 6 shows that there is a corrosion with resistivity $5 \times 10^{-6} \Omega \cdot \mathrm{m}$ between electrode $\mathrm{C}$ and electrode $\mathrm{D}$.

According to the simulation procedures described above, the potentials at the measurement electrodes are computed.

For SIE model, the relative potentials are:

$$
\begin{aligned}
& I^{\prime}=100 \mathrm{~A} \\
& U_{C D}^{\prime}=0.003418 \mathrm{~V} \\
& U_{D E}^{\prime}=0.002829 \mathrm{~V} \\
& I=100 \mathrm{~A} \\
& U_{C}=1.232704 \mathrm{~V} \\
& U_{D}=1.229286 \mathrm{~V} \\
& U_{E}=1.226460 \mathrm{~V}
\end{aligned}
$$

For DIE model, the relative potentials are:

$$
\begin{aligned}
& I_{A}=I_{F}=100 \mathrm{~A} \\
& U_{A-C}=1.232704 \mathrm{~V} \\
& U_{A-D}=1.229286 \mathrm{~V} \\
& U_{A-E}=1.226460 \mathrm{~V} \\
& U_{F-C}=1.225964 \mathrm{~V} \\
& U_{F-D}=1.229049 \mathrm{~V} \\
& U_{F-E}=1.231658 \mathrm{~V}
\end{aligned}
$$

Based on equations (4) and (8), $\rho_{5}$ and $\rho_{6}$, formation resistivities for the case of a casing joint from SIE and DIE models, respectively can be easily calculated, as shown in TABLE I.

TABLE I

$h p$ FEM simulation results

\begin{tabular}{|c|c|c|c|c|c|c|}
\hline $\begin{array}{c}\text { Formation } \\
\text { Resistivity }\end{array}$ & \multicolumn{3}{|c|}{ SIE Model } & \multicolumn{3}{c|}{ DIE Model } \\
\hline$\rho(\Omega \cdot \mathrm{m})$ & $\rho_{l}(\Omega \cdot \mathrm{m})$ & $\rho_{3}(\Omega \cdot \mathrm{m})$ & $\rho_{5}(\Omega \cdot \mathrm{m})$ & $\rho_{2}(\Omega \cdot \mathrm{m})$ & $\rho_{4}(\Omega \cdot \mathrm{m})$ & $\rho_{6}(\Omega \cdot \mathrm{m})$ \\
\hline
\end{tabular}




\begin{tabular}{|c|c|c|c|c|c|c|}
\hline 1 & 1.08832 & -0.15268 & 0.056982 & 1.102353 & 1.132804 & 1.109076 \\
\hline 10 & 11.7206 & -1.38802 & 0.530107 & 11.54158 & 12.09743 & 11.86932 \\
\hline 100 & 120.8839 & -13.7254 & 5.255496 & 119.039 & 124.3616 & 119.905 \\
\hline
\end{tabular}

TABLE II

Theoretical analysis results

\begin{tabular}{|c|c|c|c|c|c|c|}
\hline $\begin{array}{l}\text { Formation } \\
\text { Resistivity }\end{array}$ & \multicolumn{3}{|c|}{ SIE Model } & \multicolumn{3}{c|}{ DIE Model } \\
\hline$\rho_{(\Omega \cdot \mathrm{m})}$ & $\rho_{l}(\Omega \cdot \mathrm{m})$ & $\rho_{3}(\Omega \cdot \mathrm{m})$ & $\rho_{5}(\Omega \cdot \mathrm{m})$ & $\rho_{2}(\Omega \cdot \mathrm{m})$ & $\rho_{4}(\Omega \cdot \mathrm{m})$ & $\rho_{6}(\Omega \cdot \mathrm{m})$ \\
\hline 1 & 1 & -0.38 & 0.06 & 1 & 1 & 1.09 \\
\hline 10 & 10 & -3.8 & 0.6 & 10 & 10 & 10.9 \\
\hline 100 & 100 & -38 & 6 & 100 & 100 & 109 \\
\hline
\end{tabular}

\section{Compensation effect of DIE model}

In DIE model, the calculated formation resistivities $\rho_{2}$, $\rho_{4}$ and $\rho_{6}$ are very close to the assumed real formation resistivity. However, $\rho_{3}$ and $\rho_{5}$ calculated from SIE model have great errors compared with the real formation resistivity. A clear conclusion can be drawn: minor case joint or corrosion will bring great errors to SIE model, but the DIE model is insensitive to the uneven metal casing conditions such as case joints and case corrosion.

In Section "Theoretical Analysis", the errors caused by resistance variations were proved to have relationship with coefficients $K_{1}, K_{2}, h_{2}$ and $h_{3}$. In the specific conditions described above, all the coefficients were known, and the relative formation resistivities $\left(\rho_{1}\right.$ to $\left.\rho_{6}\right)$ could be estimated according to equation (17). The estimation results are shown in TABLE II. Comparing the data listed in TABLE I and TABLE II, it can be found that the $h p$-FEM simulation results have good accuracy and agrees well with the theoretical results.

To show the robustness of the DIE method, we have considered three different formation resistivities. The relative apparent resistivities we obtain for the SIE and DIE models are shown in TABLE I. In all cases we observe a superior accuracy of the DIE method with respect to the SIE method.

\section{Conclusions}

In this contribution, the working principles of SIE model and DIE model were presented. Based on theoretical analysis and self-adaptive goal oriented $h p$-FEM simulations, we provide quantitative estimates of the errors in apparent resistivity readings caused by casing joints and casing corrosion, which are typical field situations with uneven metal casing. Comparing the calculated results with the assumed real formation resistivities, we conclude that even minor casing joint or corrosion will bring great errors to SIE model, while DIE model is always immune to the affecting factors. Moreover, the results of theoretical analysis are backed up by those of numerical simulations.

\section{Acknowledgments}

The second author was partially supported under the project of the Ministry of Sciences and Innovation MTM2010-16511.

\section{References}

[1] Alpin, L. M., The method for logging in cased wells. U.S.S.R.patent 56026, 1939.

[2] Schenkel, C. J., and Morrison, H. F., "Effects of well casing on potential-field measurements using downhole current sorces," Geophys. Prosp., vol.38, no.6, pp. 663-686, Apr.1990.

[3] Kaufman, A.A., "The electrical field in the borehole with the casing," Geophysics, vol.55, no.1, pp.29-38, 1990.

[4]Kaufman, A.A et.al, "A transmission-line model for electrical logging through casing," Geophysics, vol.58, no.12, pp.1739-1747, 1993.

[5] Vail, W. B, "Through Casing Resistivity Tool TM to locate bypassed oil," The American Oil \& Gas Reporter, vol.38, no.11, pp.70-76, Nov.1995.

[6] Schenkel, C. J., and Morrison, H. F., "Electrical resistivity measurement through metal casing," Geophysics, 59, 1072-1082, 1994.

[7] Tabarovsky, L. A, Through-casing resistivity (TCR): Physics, Resolution and 3-D effects: Presented at the 35th Ann. Logging Symposium, Soc. Prof.Well Log Analysts, 1994.

[8] Zinger, B. Sh. et. al., Modeling of electrical effects of borehole casing inhomogeneities: 64th Ann. Internat. Mtg., Soc. Expl. Geophys., Expanded Abstracts, 399-402, 1994.

[9] Singer, B.Sh., Through-casing resistivity: 2-D and 3-D distortions and correction techniques: Presented at 36th Ann. Logging Symposium,Soc. Prof.Well Log Analysts, 1995.

[10] Singer, B.S. and Strack K.M. "New aspects of through-casing resistivity theory," Geophysics, vol.63, no.1, pp.52-63, Jan.1998.

[11] Sheinmann, S. M., On stabilization of electrical fields in the earth: Pricladnaya Geophyzika, 3, 3-15 (in Russian), 1947.

[12] Vail, W.B., Electronic measurement apparatus movable in a cased borehole and compensation for casing resistance differences: U.S. Patent 5075626, 1991.

[13] Chen Qing, David Pardo, Li Hong-bin, Wang Fu-rong, and Ye 
Qi-zheng. Arithmetic Method of Double-Injection-Electrode Model for Resistivity Measurement Through Metal Casing. IEEE Trans. on Geoscience\&Remote Sensing, vol.48, no.1, pp. 36-41, Jan. 2010.

[14] Nam MJ, Pardo D and Torres-Verdin C, "Simulation of DC dual-laterolog measurements in complex formations: A Fourier-series approach with nonorthogonal coordinates and self-adapting finite elements," Geophysics, vol.74, no.1, pp.E31-E43, Jan. 2009.

[15] Pardo, D., "Simulation of multifrequency borehole resistivity measurements through metal casing using a goal-oriented hp finite-element method," IEEE Trans. on Geoscience\&Remote Sensing, vol.44, no.8, pp.2125-2134, Aug. 2006.

[15] Pardo D. , Torres-Verdin C., Nam MJ., Paszynski M. and Calo VM, "Fourier series expansion in a non-orthogonal system of coordinates for the simulation of 3D alternating current borehole resistivity measurements," Computer methods in applied mechanics and engineering, vol.197, no.45-48, pp.3836-3849, 2008.

[16] Pardo D., Demkowicz L., Torres-Verdin C. and Michler C, "PML Enhanced with a self-adaptive coal-orineted hp-finite element method simulation of through casing borehole resistivity measurements" SIAM Journal on scientific computing, vol.30, no.6, pp.2948-2964, 2007.

[17] Pardo D, Garcia-Castillo LE, Demkowicz LF and Torres-Verdin C., "A two-dimensional self-adaptive hp finite element method for the characterization of waveguide discontinuities. Part II:
Goal-oriented hp-adaptivity," Computer methods in applied mechanics and engineering, vol.196, no.49-52, pp.4811-4822, 2007.

[18] Pardo D., Calo VM., Torres-Verdin C. and Nam MJ. "Fourier series expansion in a non-orthogonal system of coordinates for the simulation of 3D-DC borehole resistivity measurements," Computer methods in applied mechanics and engineering, vol.197, no.21-24, pp.1906-1925, 2008.

[19] Hongnian Wang, "Simultaneous reconstruction of geometric parameter and resistivity around borehole in horizontally stratified formation from multiarray induction logging data," IEEE Trans. on Geoscience\&Remote Sensing. Vol41, no.1, pp.81-89, 2003.

[20] Yik-Kiong Hue ,Teixeira, F.L. "Numerical Mode-Matching Method for Tilted-Coil Antennas in Cylindrically Layered Anisotropic Media With Multiple Horizontal Beds" IEEE Trans. on Geoscience\&Remote Sensing. Vol.45, no.8, pp.2451-2462, 2007.

[21] Guozhong Gao and Carlos Torres-Verdín, "Efficient Numerical Simulation of Axisymmetric Electromagnetic Induction Measurements Using a High-Order Generalized Extended Born Approximation," IEEE Trans. on Geoscience\&Remote Sensing. Vol.44, no.9, pp.2445-2453, 2006. 